\title{
Web Applications Vulnerability Management using a Quantitative Stochastic Risk Modeling Method
}

\author{
Sergiu SECHEL \\ Bucharest University of Economic Studies \\ sergiu.sechel@gmail.com
}

The aim of this research is to propose a quantitative risk modeling method that reduces the guess work and uncertainty from the vulnerability and risk assessment activities of web based applications while providing users the flexibility to assess risk according to their risk appetite and tolerance with a high degree of assurance. The research method is based on the research done by the OWASP Foundation on this subject but their risk rating methodology needed debugging and updates in different in key areas that are presented in this paper. The modified risk modeling method uses Monte Carlo simulations to model risk characteristics that can't be determined without guess work and it was tested in vulnerability assessment activities on real production systems and in theory by assigning discrete uniform assumptions to all risk characteristics (risk attributes) and evaluate the results after 1.5 million rounds of Monte Carlo simulations.

Keywords: Vulnerabilities, Quantitative Risk, Web Applications, Monte Carlo, Stochastic Systems, Cybersecurity

1 Introduction

Web based business applications are becoming the preferred way of conducting day to day business activities as part of a digital business model. As such, there is a growing concern related to data breaches that affected major digital businesses in the past years. An updated list of the world biggest data breaches is available at World's Biggest Data Breaches [1]. An cybersecurity report published by Ernst \& Young in 2017 shows that poor risk assessment and management practices are a big cause for data breaches and cyberattacks: "With the quality of reporting being so low, it is no surprise that $52 \%$ of responders think their boards are not fully knowledgeable about the risks the organization is taking and the measures that are in place. In other words, our survey suggests that about half of all boards."'[2] Most organizations are using superficial qualitative risk management methodologies when they are assessing IT related risks using formulas that require a lot of guess work and allow for high level of uncertainty with low assurance. The aim of this research is to propose a quantitative risk modeling method that reduces the guess work and uncertainty from the vulnerability and risk assessment activities of web based applications while providing users the flexibility to assess risk according to their risk appetite and tolerance with a high degree of assurance. The research method is based on the research done by the OWASP Foundation on this subject but their risk rating methodology needed debugging and updates in different in key areas that are presented in this paper. The modified risk modeling method uses Monte Carlo simulations to model risk characteristics that can't be determined without guess work and it was tested in vulnerability assessment activities on real production systems and in theory by assigning discrete uniform assumptions to all risk characteristics (risk attributes) and evaluate the results after 1.5 million rounds of Monte Carlo simulations.

\section{Methodology}

We utilized the OWASP Risk Rating Methodology [3] as a starting point for the risk modeling activities because is widespread use among web application cybersecurity specialists. The OWASP [4] is a community driven not for profit group that aims to improve the security of web based applications, and they periodically publish the classification report OWASP Top 10 Most Critical Web Application Security Risks, summarized in Table 1. 
Table 1. OWASP Top 10 Most Critical Web Application Security Risks Summary

\begin{tabular}{|c|c|}
\hline OWASP ID & Description \\
\hline $\begin{array}{c}\mathrm{A} 1 \\
\text { Injection }\end{array}$ & $\begin{array}{l}\text { Injection flaws, such as SQL, OS, XXE, and LDAP injection occur when } \\
\text { untrusted data is sent to an interpreter as part of a command or query. The } \\
\text { attacker's hostile data can trick the interpreter into executing unintended } \\
\text { commands or accessing data without proper authorization. }\end{array}$ \\
\hline $\begin{array}{l}\text { A2 } \\
\text { Broken Au- } \\
\text { thentication } \\
\text { and Session } \\
\text { Management }\end{array}$ & $\begin{array}{l}\text { Application functions related to authentication and session management are } \\
\text { often implemented incorrectly, allowing attackers to compromise passwords, } \\
\text { keys, or session tokens, or to exploit other implementation flaws to assume } \\
\text { other users' identities (temporarily or permanently). }\end{array}$ \\
\hline $\begin{array}{l}\text { A3 } \\
\text { Cross-Site } \\
\text { Scripting } \\
\text { (XSS) }\end{array}$ & $\begin{array}{l}\text { XSS flaws occur whenever an application includes untrusted data in a new } \\
\text { web page without proper validation or escaping, or updates an existing web } \\
\text { page with user supplied data using a browser API that can create JavaScript. } \\
\text { XSS allows attackers to execute scripts in the victim's browser which can } \\
\text { hijack user sessions, deface web sites, or redirect the user to malicious sites. }\end{array}$ \\
\hline $\begin{array}{l}\text { A4 } \\
\text { Broken Ac- } \\
\text { cess } \\
\text { Control }\end{array}$ & $\begin{array}{l}\text { Restrictions on what authenticated users are allowed to do are not properly } \\
\text { enforced. Attackers can exploit these flaws to access unauthorized function- } \\
\text { ality and/or data, such as access other users' accounts, view sensitive files, } \\
\text { modify other users' data, change access rights, etc. }\end{array}$ \\
\hline $\begin{array}{l}\text { A5 } \\
\text { Security } \\
\text { Misconfigu- } \\
\text { ration }\end{array}$ & $\begin{array}{l}\text { Good security requires having a secure configuration defined and deployed } \\
\text { for the application, frameworks, application server, web server, database } \\
\text { server, platform, etc. Secure settings should be defined, implemented, and } \\
\text { maintained, as defaults are often insecure. Additionally, software should be } \\
\text { kept up to date. }\end{array}$ \\
\hline $\begin{array}{l}\text { A6 } \\
\text { Sensitive } \\
\text { Data } \\
\text { Exposure }\end{array}$ & $\begin{array}{l}\text { Many web applications and APIs do not properly protect sensitive data, such } \\
\text { as financial, healthcare, and PII. Attackers may steal or modify such weakly } \\
\text { protected data to conduct credit card fraud, identity theft, or other crimes. } \\
\text { Sensitive data deserves extra protection such as encryption at rest or in } \\
\text { transit, as well as special precautions when exchanged with the browser. }\end{array}$ \\
\hline $\begin{array}{l}\text { A7 } \\
\text { Insufficient } \\
\text { Attack Pro- } \\
\text { tection }\end{array}$ & $\begin{array}{l}\text { The majority of applications and APIs lack the basic ability to detect, pre- } \\
\text { vent, and respond to both manual and automated attacks. Attack protection } \\
\text { goes far beyond basic input validation and involves automatically detecting, } \\
\text { logging, responding, and even blocking exploit attempts. Application owners } \\
\text { also need to be able to deploy patches quickly to protect against attacks. }\end{array}$ \\
\hline $\begin{array}{l}\text { A8 } \\
\text { Cross-Site } \\
\text { Request For- } \\
\text { gery (CSRF) }\end{array}$ & $\begin{array}{l}\text { A CSRF attack forces a logged-on victim's browser to send a forged HTTP } \\
\text { request, including the victim's session cookie and any other automatically } \\
\text { included authentication information, to a vulnerable web application. Such } \\
\text { an attack allows the attacker to force a victim's browser to generate requests } \\
\text { the vulnerable application thinks are legitimate requests from the victim. }\end{array}$ \\
\hline $\begin{array}{c}\text { A9 } \\
\text { Using Com- } \\
\text { ponents with } \\
\text { Known } \\
\text { Vulnerabili- } \\
\text { ties } \\
\end{array}$ & $\begin{array}{l}\text { Components, such as libraries, frameworks, and other software modules, run } \\
\text { with the same privileges as the application. If a vulnerable component is ex- } \\
\text { ploited, such an attack can facilitate serious data loss or server takeover. Ap- } \\
\text { plications and APIs using components with known vulnerabilities may un- } \\
\text { dermine application defenses and enable various attacks and impacts. }\end{array}$ \\
\hline $\begin{array}{l}\text { A10 } \\
\text { Under pro- } \\
\text { tected API's }\end{array}$ & $\begin{array}{l}\text { Modern applications often involve rich client applications and APIs, such as } \\
\text { JavaScript in the browser and mobile applications that connect to an API of } \\
\text { some kind (SOAP/XML, REST/JSON, RPC, GWT, etc.). These APIs are } \\
\text { often unprotected and contain numerous vulnerabilities. }\end{array}$ \\
\hline
\end{tabular}


The OWASP Risk Rating Methodology assumes that a discovered vulnerability will impact a web application on 2 main areas: technical and business; and to determine the probability that a vulnerability will be exploited it measures the likelihood of an attack by determining the vulnerability profile (vulnerability assessment) and the threat agent profile (what type of agent will use that vulnerability in an attack. By design the OWASP Risk Rating Methodology ask the cybersecurity specialist do determine by itself or with help of peers how a discovered vulnerability should score on each of the 4 risk subcomponents. Each of the 4 risk subcomponents has 4 attributes categories that are presented in the Table 7 through Table 22, and each attributes category has 1-9 scored scale that measures the severity of that attribute. For each attributes category the global OWASP community collectively determined how many positions to be measured. Each category attribute receives a score from $1(0.125)$ to 9 (1.125), and then the risk subcomponents and risk components are quantitatively determined using the formulas from Table 4 while the qualitative scale is presented in Table 5 and the qualitative risk matrix is presented in Table 7. For each attribute category the OWASP community determined the measures descriptions and although arguments can be made for and against how each measures description was chosen in the end the OWASP community reached a consensus. The risk modeling methodology used in this research is based on OWASP Risk Rating Methodology (presented above) with the following modifications to eliminate inherited errors and bias:

- the scale score presented in Table 2 uses only the 1-9 scored scale, while the original OWASP Risk Rating Methodology uses a 0-9 scored scale, which in my opinion is not needed since the discovery of a vulnerability can't have a 0 score from any risk methodology perspective;

- the classification rational between the 4 risk subcomponents is changed from the OWASP Risk Rating Methodology by assuming that the Threat Agent Profile and the Business Impact subcomponent are difficult to determine for a discovered vulnerability without bias, Table 3 ;

- all the attributes that are difficult to determine through human rational thinking and experience are determined using Monte Carlo simulations using the Oracle Crystal Ball software [5].

Table 2. Risk modeling score scale

\begin{tabular}{|c|l|c|l|}
\hline Scale & $\begin{array}{l}\text { Score } \\
(\mathbf{0 . 1 2 5} \text { increments })\end{array}$ & Scale & $\begin{array}{l}\text { Score } \\
(\mathbf{0 . 1 2 5} \text { increments })\end{array}$ \\
\hline 1 & 0.125 & 6 & 0.750 \\
\hline 2 & 0.250 & 7 & 0.875 \\
\hline 3 & 0.375 & 8 & 1.000 \\
\hline 4 & 0.500 & 9 & 1.125 \\
\hline 5 & 0.625 & & \\
\hline
\end{tabular}

Table 3. Risk subcomponents ease of classification without stochastic modeling

\begin{tabular}{|c|l|l|l|}
\hline No. & \multirow{2}{*}{ Risk Components } & Subcomponents & $\begin{array}{l}\text { Ease of classification without sto- } \\
\text { chastic modeling }\end{array}$ \\
\hline 1 & \multirow{2}{*}{ Likelihood } & Threat Agent Profile & Difficult to determine without bias \\
\cline { 4 - 4 } 2 & & Vulnerability Profile & Easy to determine \\
\hline 3 & \multirow{2}{*}{ Impact } & Technical Impact & Easy to determine \\
\cline { 3 - 4 } 4 & & Business Impact & Difficult to determine without bias \\
\hline
\end{tabular}


Table 4. Risk modeling formulas

\begin{tabular}{|l|l|l|}
\hline No. & Description & Formula \\
\hline 1 & Likelihood Score & Threat Agent Profile + Vulnerability Profile \\
\hline 2 & Impact Score & Technical Impact + Business Impact \\
\hline 3 & Risk Score & (Likelihood Score * Impact Score)/8 \\
\hline
\end{tabular}

Table 5. Risk components qualitative classification

\begin{tabular}{|l|l|}
\hline Likelihood and Impact Levels & Risk Classification \\
\hline 0 to $<3$ & LOW \\
\hline 3 to $<6$ & MEDIUM \\
\hline 6 to 9 & HIGH \\
\hline
\end{tabular}

Table 6. Risk score qualitative classification matrix

\begin{tabular}{|c|r|c|c|c|}
\hline \multicolumn{5}{|c|}{ Risk Category Matrix } \\
\hline \multirow{3}{*}{ Impact } & HIGH & Medium & High & High \\
\cline { 2 - 5 } & MEDIUM & Low & Medium & High \\
\cline { 2 - 5 } & LOW & Low & Low & Medium \\
\cline { 2 - 5 } & & LOW & MEDIUM & HIGH \\
\hline & \multicolumn{4}{|c|}{ Likelihood } \\
\hline
\end{tabular}

Each discovered web application vulnerability $\quad 7,8,9,10,11,12,13,14,15,1,6,17,18,19,20,21$, is measured against the following attributes categories presented below in the Table

Table 7. Attribute Category - Threat Agent Profile (Skill Level)

\begin{tabular}{|c|l|l|}
\hline Scale & $\begin{array}{l}\text { Threat Agent Profile - Skill Level } \\
\text { How technically skilled is this group of threat agents? }\end{array}$ & $\begin{array}{l}\text { Score } \\
\mathbf{0 . 1 2 5} \text { increments })\end{array}$ \\
\hline 1 & No Technical Skills & 0.125 \\
\hline 3 & Limited Technical Skills & 0.375 \\
\hline 5 & Advanced Technical Skills & 0.625 \\
\hline 6 & Specific Programming Skills & 0.750 \\
\hline 9 & Cybersecurity Penetration Testing Skills & 1.125 \\
\hline
\end{tabular}

Table 8. Attribute Category - Threat Agent Profile (Motive)

\begin{tabular}{|c|l|l|}
\hline Scale & $\begin{array}{l}\text { Threat Agent Profile - Motive } \\
\text { How motivated is this group of threat agents to find and exploit this vulnerability? }\end{array}$ & $\begin{array}{l}\text { Score } \\
\mathbf{0 . 1 2 5} \text { increments) }\end{array}$ \\
\hline 1 & Not Financially Motivated & 0.125 \\
\hline 4 & Small Financial Rewards & 0.500 \\
\hline 9 & High Financial Rewards & 1.125 \\
\hline
\end{tabular}

Table 9. Attribute Category - Threat Agent Profile (Opportunity)

\begin{tabular}{|c|l|l|}
\hline Scale & $\begin{array}{l}\text { Threat Agent Profile - Opportunity } \\
\text { What resources and opportunities are required for this group of threat agents to find and ex- } \\
\text { ploit this vulnerability? }\end{array}$ & $\begin{array}{l}\text { Score } \\
\mathbf{( 0 . 1 2 5} \text { increments) }\end{array}$ \\
\hline 1 & Full Access or Expensive Resources Required & 0.125 \\
\hline 4 & Special Access or Resources Required & 0.500 \\
\hline 7 & Limited Access or Resources Required & 0.875 \\
\hline 9 & No Access or Resources Required & 1.125 \\
\hline
\end{tabular}


Table 10. Attribute Category - Threat Agent Profile (Relationship)

\begin{tabular}{|c|l|l|}
\hline Scale & $\begin{array}{l}\text { Threat Agent Profile - Relationship } \\
\text { How large is this group of threat agents in relationship to the target system? }\end{array}$ & $\begin{array}{l}\text { Score } \\
\mathbf{( 0 . 1 2 5} \text { increments) }\end{array}$ \\
\hline 2 & Internal Developers or System Administrators & 0.125 \\
\hline 4 & Intranet Users & 0.500 \\
\hline 5 & Partners & 0.625 \\
\hline 6 & Authenticated Users & 0.750 \\
\hline 9 & Anonymous Internet Users & 1.125 \\
\hline
\end{tabular}

Table 11. Attribute Category - Vulnerability Profile (Ease of discovery)

\begin{tabular}{|c|l|l|}
\hline Scale & $\begin{array}{l}\text { Vulnerability Profile - Ease of Discovery } \\
\text { How easy is it for this group of threat agents to discover this vulnerability? }\end{array}$ & $\begin{array}{l}\text { Score } \\
\mathbf{( 0 . 1 2 5} \text { increments })\end{array}$ \\
\hline 2 & Internal Developers or System Administrators & 0.250 \\
\hline 4 & Intranet Users & 0.500 \\
\hline 5 & Partners & 0.625 \\
\hline 6 & Authenticated Users & 0.750 \\
\hline 9 & Anonymous Internet Users & 1.125 \\
\hline
\end{tabular}

Table 12. Attribute Category - Vulnerability Profile (Ease of exploitation)

\begin{tabular}{|c|l|l|}
\hline Scale & $\begin{array}{l}\text { Vulnerability Profile - Ease of Exploitation } \\
\text { How easy is it for this group of threat agents to actually exploit this vulnerability? }\end{array}$ & $\begin{array}{l}\text { Score } \\
\text { (0.125 increments) }\end{array}$ \\
\hline 1 & Practically impossible & 0.125 \\
\hline 3 & Difficult & 0.375 \\
\hline 7 & Easy & 0.875 \\
\hline 9 & Automated tools available & 1.125 \\
\hline
\end{tabular}

Table 13. Attribute Category - Vulnerability Profile (Awareness)

\begin{tabular}{|c|l|l|}
\hline Scale & $\begin{array}{l}\text { Vulnerability Profile - Awareness } \\
\text { How well known is this vulnerability to this group of threat agents? }\end{array}$ & $\begin{array}{l}\text { Score } \\
\text { (0.125 increments) }\end{array}$ \\
\hline 1 & Theoretical & 0.125 \\
\hline 3 & Difficult & 0.375 \\
\hline 5 & Easy & 0.625 \\
\hline 9 & Automated tools available & 1.125 \\
\hline
\end{tabular}

Table 14. Attribute Category - Vulnerability Profile (Intrusion detection)

\begin{tabular}{|c|l|l|}
\hline Scale & $\begin{array}{l}\text { Vulnerability Profile - Intrusion Detection } \\
\text { How likely is an exploit to be detected? }\end{array}$ & $\begin{array}{l}\text { Score } \\
\text { (0.125 increments })\end{array}$ \\
\hline 1 & Active detection in application & 0.125 \\
\hline 3 & Logged and reviewed & 0.375 \\
\hline 8 & Logged without review & 1.000 \\
\hline 9 & Not logged & 1.125 \\
\hline
\end{tabular}

Table 15. Attribute Category - Technical Impact (Loss of confidentiality)

\begin{tabular}{|c|l|l|}
\hline Scale & $\begin{array}{l}\text { Technical Impact - Loss of Confidentiality } \\
\text { How much data could be disclosed and how sensitive is it? }\end{array}$ & $\begin{array}{l}\text { Score } \\
\mathbf{0 . 1 2 5} \text { increments })\end{array}$ \\
\hline 3 & Minimal non-sensitive data disclosed & 0.375 \\
\hline 5 & $\begin{array}{l}\text { Minimal critical data disclosed / Extensive non-sensitive data dis- } \\
\text { closed }\end{array}$ & 0.625 \\
\hline 6 & Extensive critical data disclosed & 0.750 \\
\hline 9 & Full data disclosed & 1.125 \\
\hline
\end{tabular}


Table 16. Attribute Category - Technical Impact (Loss of integrity)

\begin{tabular}{|c|l|l|}
\hline Scale & $\begin{array}{l}\text { Technical Impact - Loss of Integrity } \\
\text { How much data could be corrupted and how damaged is it? }\end{array}$ & $\begin{array}{l}\text { Score } \\
\mathbf{( 0 . 1 2 5} \text { increments) }\end{array}$ \\
\hline 1 & Minimal data corruption & 0.125 \\
\hline 3 & Low data corruption & 0.375 \\
\hline 5 & Medium data corruption & 0.625 \\
\hline 7 & Extensive data corruption & 0.875 \\
\hline 9 & Full data corruption & 1.125 \\
\hline
\end{tabular}

Table 17. Attribute Category - Technical Impact (Loss of availability)

\begin{tabular}{|c|l|l|}
\hline Scale & $\begin{array}{l}\text { Technical Impact - Loss of Availability } \\
\text { How much service could be lost and how vital is it? }\end{array}$ & $\begin{array}{l}\text { Score } \\
\text { (0.125 increments) }\end{array}$ \\
\hline 1 & Minimal secondary services interrupted & 0.125 \\
\hline 5 & $\begin{array}{l}\text { Minimal primary services interrupted, extensive secondary ser- } \\
\text { vices interrupted }\end{array}$ & 0.625 \\
\hline 7 & Extensive primary services interrupted & 0.875 \\
\hline 9 & All services completely lost & 1.125 \\
\hline
\end{tabular}

Table 18. Attribute Category - Technical Impact (Loss of accountability)

\begin{tabular}{|c|l|l|}
\hline Scale & $\begin{array}{l}\text { Technical Impact - Loss of accountability } \\
\text { Are the threat agent's actions traceable to an individual? }\end{array}$ & $\begin{array}{l}\text { Score } \\
\mathbf{( 0 . 1 2 5} \text { increments })\end{array}$ \\
\hline 1 & Fully traceable & 0.125 \\
\hline 7 & Possibly traceable & 0.875 \\
\hline 9 & Completely anonymous & 1.125 \\
\hline
\end{tabular}

Table 19. Attribute Category - Business Impact (Financial Impact)

\begin{tabular}{|c|l|l|}
\hline Scale & $\begin{array}{l}\text { Business Impact - Financial Damage } \\
\text { How much financial damage will result from an exploit? }\end{array}$ & $\begin{array}{l}\text { Score } \\
\text { (0.125 increments) }\end{array}$ \\
\hline 1 & Less than the cost to fix the vulnerability & 0.125 \\
\hline 3 & Minor effect on annual revenue & 0.375 \\
\hline 7 & Significant effect on annual revenue & 0.875 \\
\hline 9 & Bankruptcy & 1.125 \\
\hline
\end{tabular}

Table 20. Attribute Category - Business Impact (Reputation Damage)

\begin{tabular}{|c|l|l|}
\hline Scale & $\begin{array}{l}\text { Business Impact - Reputation Damage } \\
\text { Would an exploit result in reputation damage that would harm the business? }\end{array}$ & $\begin{array}{l}\text { Score } \\
\text { (0.125 increments) }\end{array}$ \\
\hline 1 & Minimal damage & 0.125 \\
\hline 4 & Loss of major accounts & 0.500 \\
\hline 5 & Loss of goodwill & 0.625 \\
\hline 9 & Brand damage & 1.125 \\
\hline
\end{tabular}

Table 21. Attribute Category - Business Impact (Non-compliance)

\begin{tabular}{|c|l|l|}
\hline Scale & $\begin{array}{l}\text { Business Impact - Non-Compliance } \\
\text { How much exposure does non-compliance introduce? }\end{array}$ & $\begin{array}{l}\text { Score } \\
\mathbf{( 0 . 1 2 5} \text { increments) }\end{array}$ \\
\hline 2 & Minor violation & 0.250 \\
\hline 5 & Significant violation & 0.625 \\
\hline 7 & Clear violation & 0.875 \\
\hline
\end{tabular}


Table 22. Attribute Category - Business Impact (Privacy violation)

\begin{tabular}{|c|l|l|}
\hline Scale & $\begin{array}{l}\text { Business Impact - Privacy Violation } \\
\text { How much personally identifiable information could be disclosed? }\end{array}$ & $\begin{array}{l}\text { Score } \\
\mathbf{0 . 1 2 5} \text { increments })\end{array}$ \\
\hline 3 & One individual & 0.375 \\
\hline 5 & Hundreds of people & 0.625 \\
\hline 7 & Thousands of people & 0.875 \\
\hline 9 & Millions of people & 1.125 \\
\hline
\end{tabular}

\section{Methodology validation}

The methodology and risk modeling formulas were verified using the Oracle Crystal Ball software by assigning to each attribute category a discrete uniform assumption between the declared measures with an equal probability for each measure. For example using the measures in Table 22, the discrete uniform assumption is that there is a 0.25 (there a 4 measures, $0.25 \times 4=1$ ) (the probability scale is between 0 and 1) chance that one of the $[0.375,0.625,0.875,1.125]$ will happen during a Monte Carlo simulation round. The validation exercise tested the validity of the formulas presented in Table 4 with 1.500 .000 rounds of Monte Carlo simulations. The exercise results are presented in Table 23, with the distribution graph presented in Fig.1. To de- termine which attribute had the greatest impact on the results of the validation exercise a sensitivity analysis was performed with the results presented in Fig.2. From the sensitivity analysis results a preliminary conclusion can be drawn that from the top 8 attributes that have the highest impact on the exercise results only 3(motive, opportunity and financial damage) are considered to be difficult to be measured without Monte Carlo simulations or other stochastic modeling methods while the other 5 can be measured using prior knowledge and experience. The validation exercise results measured a minimum risk score of 0.492 and a maximum score of 8.898 with a mean of 3.264 and a mean standard error of 0.0008 which shows that in theory the risk model fits the declared scale of 0 to 9 for risk classification.

Table 23. Methodology validation results using 1.500.000 rounds of Monte Carlo simulations

\begin{tabular}{|l|r|l|r|}
\hline Statistics & Risk Score & Percentiles & Risk Score \\
\hline Trials & 1500000 & $0 \%$ & 0.4922 \\
\hline Base Case & 0.0000 & $5 \%$ & 1.8594 \\
\hline Mean & 3.2641 & $10 \%$ & 2.1191 \\
\hline Median & 3.1992 & $15 \%$ & 2.3125 \\
\hline Mode & 3.2813 & $20 \%$ & 2.4609 \\
\hline Standard Deviation & 0.9262 & $25 \%$ & 2.6016 \\
\hline Variance & 0.8578 & $30 \%$ & 2.7344 \\
\hline Skewness & 0.3991 & $35 \%$ & 2.8477 \\
\hline Kurtosis & 3.10 & $40 \%$ & 2.9648 \\
\hline Coefficient of Variation & 0.2837 & $45 \%$ & 3.0938 \\
\hline Minimum & 0.4922 & $50 \%$ & 3.1992 \\
\hline Maximum & 8.8984 & $55 \%$ & 3.3203 \\
\hline Range Width & 8.4063 & $60 \%$ & 3.4434 \\
\hline Mean Std. Error & 0.0008 & $65 \%$ & 3.5801 \\
\hline & & $70 \%$ & 3.6953 \\
\hline & & $75 \%$ & 3.8594 \\
\hline & & $80 \%$ & 4.0332 \\
\hline & & $85 \%$ & 4.2227 \\
\hline
\end{tabular}




\begin{tabular}{|l|l|l|r|}
\hline & & $90 \%$ & 4.4922 \\
\hline & & $95 \%$ & 4.8809 \\
\hline & & $100 \%$ & 8.8984 \\
\hline
\end{tabular}

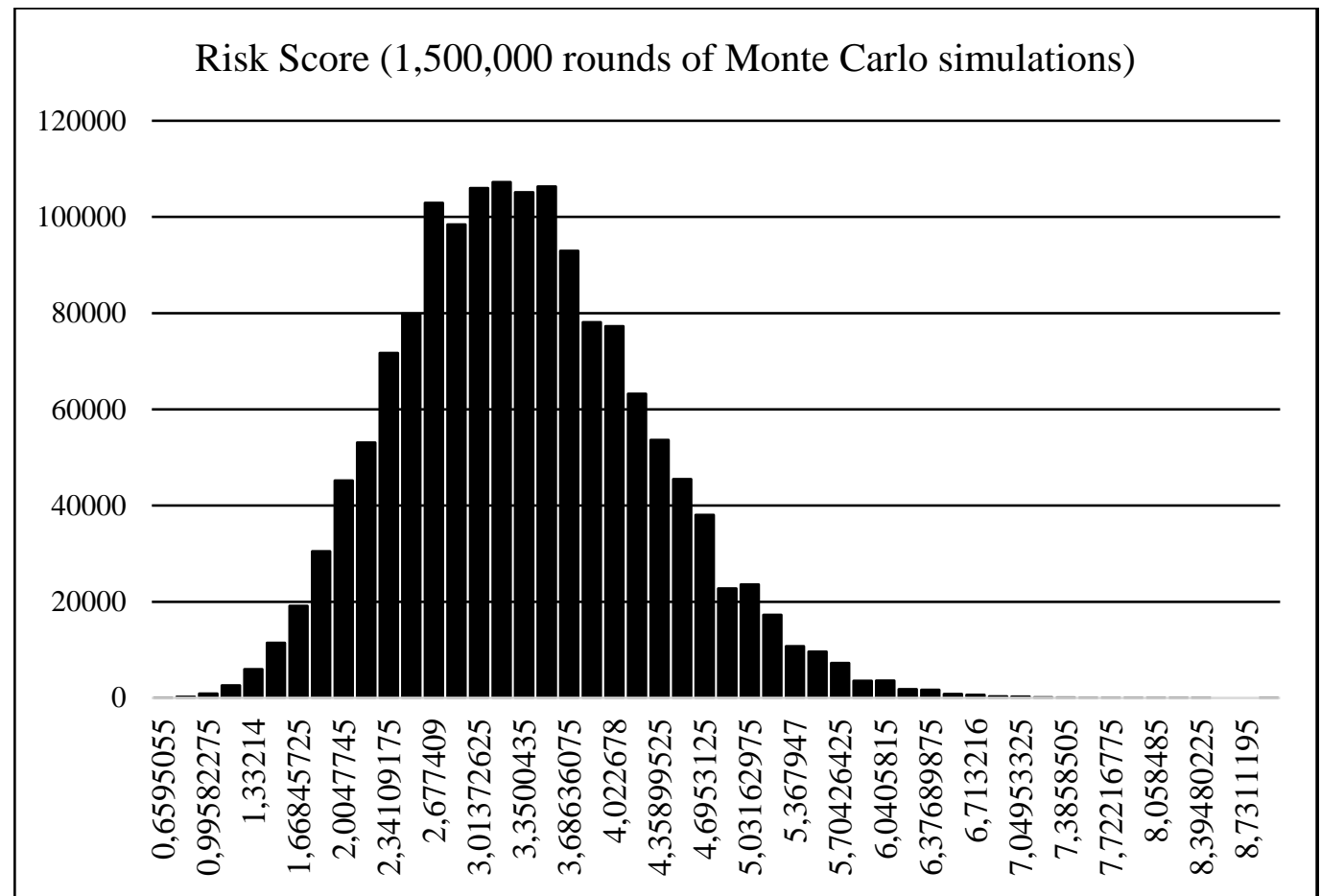

Fig. 1. Methodology validation distribution graph

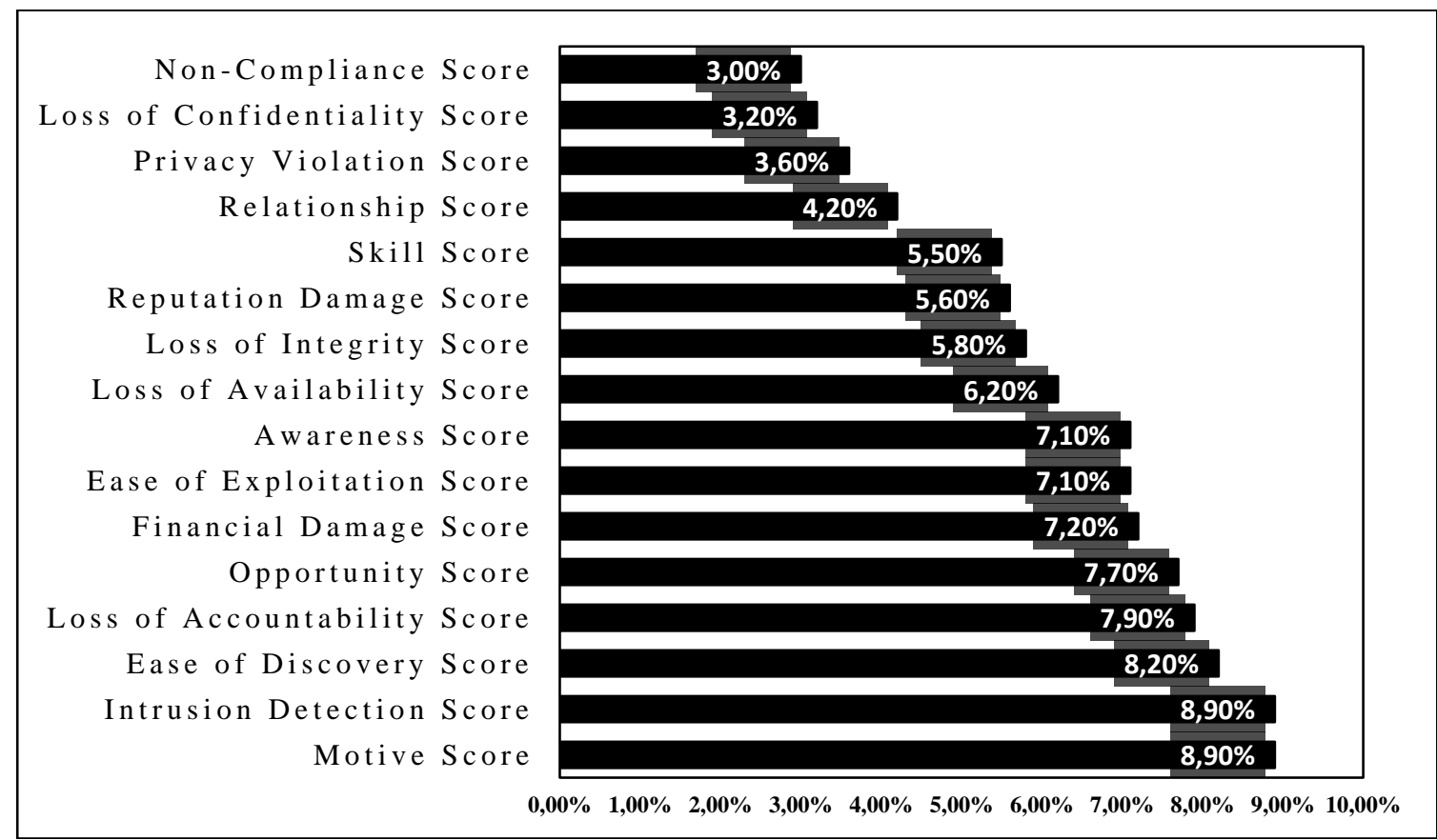

Fig. 2. Methodology validation risk sensitivity chart

\section{Experiments and results}

The risk modeling methodology was used to evaluate the risk of 3 vulnerabilities in 3 dif- ferent web based business applications implemented in production by 3 different entities. The vulnerabilities were discovered by performing vulnerability assessment services and 
penetration testing activities for each of the 3 entities in the past 6 months. The automated tool Acunetix Web Vulnerability Scanner [6] was used to perform initial vulnerability discovery and Kali Linux [7] with Metasploit [8] was used to manually validate in what circumstances each vulnerability can be exploited. The assumptions presented in Table 24 were used to determine which of the attribute categories can be determined accurately using available data and which needed Monte Carlo simulations to determine a correct risk score. For all 3 vulnerabilities 12 attribute categories were accurately determine while 6 attribute categories needed Monte Carlo simulations to determine the correct risk score. In Table 24 all attribute categories marked with M.C. were calculated using Monte Carlo (M.C.) simulations.

Table 24. The risk score template that was used for the risk modeling experiments

\begin{tabular}{|c|l|c|l|c|}
\hline $\begin{array}{c}\text { Risk } \\
\text { Component }\end{array}$ & Risk Subcomponent & Score & Risk Subcomponent & Score \\
\hline $\begin{array}{c}\text { Threat Actor } \\
\text { Profile }\end{array}$ & Skill Level & M.C. & Motive & M.C. \\
\hline $\begin{array}{c}\text { Vulnerability } \\
\text { Profile }\end{array}$ & Ease of discovery & 0 & Relationship & 0 \\
\hline Technical & Losareness & 0 & Ease of exploit & 0 \\
Impact & Loss of availability & 0 & Loss of integrity & 0 \\
\hline Business & Financial damage & M.C. & Reputation damage & M.C. \\
Impact & Non-compliance & M.C. & Privacy violation & M.C. \\
\hline
\end{tabular}

\subsection{Case 1 - Electronic Banking System -} Time Based SQL Injection Vulnerability In the web based portal for an electronic banking system a time based SQL Injection vulnerability was discovered and validated using the tool SQLMAP [9]. SQL injection is a vulnerability that allows an attacker to alter backend SQL statements by manipulating the user input. An SQL injection occurs when web applications accept user input that is directly placed into a SQL statement and doesn't properly filter out dangerous characters [10]. The vulnerability was successfully exploited and the client's database was extracted from the electronic banking system portal. In the Table 25 all the known assumptions for the attributes categories were determined and then using Oracle Crystal Ball we calculated the risk profile for the time based SQL injection vulnerability. The mean impact score is 5.21 (MEDIUM), the mean likelihood score is 6.91 (HIGH) and the mean risk score is 4.50 (MEDIUM), as presented in the Table 26. Although the quantitative analysis calculated a mean risk score of 4.50 , using the percentiles assurance values from Table 27 the organization can eliminate or keep a level of uncertainty depending on their risk appetite. In this case the organization had a very low risk appetite given the importance of their electronic banking system to their business model and business reputation and they wanted $100 \%$ assurance, meaning that the vulnerability risk score was officially 6.25 (HIGH) with an impact score of $6.25(\mathrm{HIGH})$ and a likelihood score of 8.00 (HIGH). The organization immediately allocated the resources, time and budget to repair the vulnerability. 
Table 25. Case 1 - Risk modeling assumptions

\begin{tabular}{|c|l|l|l|c|}
\hline $\begin{array}{c}\text { Risk } \\
\text { Component }\end{array}$ & Risk Subcomponent & Score & Risk Subcomponent & Score \\
\hline Threat Actor & Skill Level & M.C. & Motive & M.C. \\
Profile & Opportunity & 1.125 & Relationship & 1.125 \\
\hline Vulnerability & Ease of discovery & 1.125 & Ease of exploit & 1.125 \\
Profile & Awareness & 1.125 & Intrusion detection & 0.125 \\
\hline Technical & Loss of confidentiality & 1.125 & Loss of integrity & 0.125 \\
Impact & Loss of availability & 0.125 & Loss of accountability & 0.875 \\
\hline Business & Financial damage & M.C. & Reputation damage & M.C. \\
Impact & Non-compliance & 0.875 & Privacy violation & 0.875 \\
\hline
\end{tabular}

Table 26. Case 1 - Risk modeling results

\begin{tabular}{|l|l|l|l|}
\hline Statistics & Impact Score & Likelihood Score & Risk Score \\
\hline Trials & 100000 & 100000 & 100000 \\
\hline Base Case & 4.0000 & 5.7500 & 2.8750 \\
\hline Mean & 5.2182 & 6.9139 & 4.5096 \\
\hline Median & 5.2500 & 7.0000 & 4.4922 \\
\hline Mode & 5.2500 & 7.0000 & 4.5938 \\
\hline Standard Deviation & 0.5330 & 0.5268 & 0.5747 \\
\hline Variance & 0.2841 & 0.2776 & 0.3303 \\
\hline Skewness & 0.0757 & 0.1847 & 0.2505 \\
\hline Kurtosis & 2.29 & 2.30 & 2.69 \\
\hline Coefficient of Variation & 0.1021 & 0.0762 & 0.1274 \\
\hline Minimum & 4.2500 & 6.0000 & 3.1875 \\
\hline Maximum & 6.2500 & 8.0000 & 6.2500 \\
\hline Range Width & 2.0000 & 2.0000 & 3.0625 \\
\hline Mean Std. Error & 0.0017 & 0.0017 & 0.0018 \\
\hline
\end{tabular}

Table 27. Case 1 - Risk modeling assurance table (risk appetite/tolerance)

\begin{tabular}{|l|l|l|l|}
\hline Percentiles & Impact Score & Likelihood Score & Risk Score \\
\hline $0 \%$ & 4.2500 & 6.0000 & 3.1875 \\
\hline $5 \%$ & 4.2500 & 6.0000 & 3.6133 \\
\hline $10 \%$ & 4.5000 & 6.2500 & 3.7500 \\
\hline $15 \%$ & 4.6250 & 6.3750 & 3.9063 \\
\hline $20 \%$ & 4.7500 & 6.3750 & 3.9844 \\
\hline $25 \%$ & 4.8750 & 6.5000 & 4.0820 \\
\hline $30 \%$ & 4.8750 & 6.6250 & 4.1563 \\
\hline $35 \%$ & 5.0000 & 6.6250 & 4.2656 \\
\hline $40 \%$ & 5.0000 & 6.6250 & 4.3359 \\
\hline $45 \%$ & 5.2500 & 6.8750 & 4.3828 \\
\hline $50 \%$ & 5.2500 & 7.0000 & 4.4922 \\
\hline $55 \%$ & 5.2500 & 7.0000 & 4.5547 \\
\hline $60 \%$ & 5.3750 & 7.0000 & 4.6250 \\
\hline $65 \%$ & 5.5000 & 7.0000 & 4.7031 \\
\hline $70 \%$ & 5.5000 & 7.2500 & 4.8125 \\
\hline
\end{tabular}




\begin{tabular}{|l|l|l|l|}
\hline $75 \%$ & 5.5000 & 7.3750 & 4.9219 \\
\hline $80 \%$ & 5.6250 & 7.3750 & 5.0000 \\
\hline $85 \%$ & 5.7500 & 7.5000 & 5.1563 \\
\hline $90 \%$ & 6.0000 & 7.6250 & 5.2500 \\
\hline $95 \%$ & 6.2500 & 8.0000 & 5.4805 \\
\hline $100 \%$ & 6.2500 & 8.0000 & 6.2500 \\
\hline
\end{tabular}

4.2 Case 2 - Supply Chain Ordering System - Joomla! Core Remote Code Execution

In the web based application for a supply chain ordering system a Joomla! Core Remote Code Execution (1.5.0 - 3.4.5) vulnerability was discovered and validated using various Metasploit modules. Joomla! is prone to a remote code execution vulnerability because it fails to sufficiently sanitize user-supplied input. Successful exploitation may allow attackers to execute arbitrary commands with the privileges of the user running the application, to compromise the application or the underlying database, to access or modify data or to compromise a vulnerable system. [11] In the Table 28 all the known assumptions for the attributes categories were determined and then using Oracle Crystal Ball we calculated the risk profile for the Joomla core remote code execution vulnerability. The mean impact score is $6.31(\mathrm{HIGH})$, the mean likelihood score is 7.03 (HIGH) and the mean risk score is 5.57 (MEDIUM), as presented in the Table 29. Although the quantitative analysis calculated a mean risk score of 5.57, using the percentiles assurance values from Table 30 the organization can eliminate or keep a level of uncertainty depending on their risk appetite. In this case the organization had a low risk appetite given the importance of their supply chain ordering system to their business model and business reputation and they wanted $80 \%$ assurance, meaning that the vulnerability risk score was officially 6.10 (HIGH) with an impact score of $6.75(\mathrm{HIGH})$ and a likelihood score of 7.50 (HIGH). The results were presented to the organization's management who allocated the resources, time and budget to repair the vulnerability.

Table 28. Case 2 - Risk modeling assumptions

\begin{tabular}{|c|l|c|l|c|}
\hline $\begin{array}{c}\text { Risk } \\
\text { Component }\end{array}$ & Risk Subcomponent & Score & Risk Subcomponent & Score \\
\hline $\begin{array}{c}\text { Threat Actor } \\
\text { Profile }\end{array}$ & Skill Level & M.C. & Motive & M.C. \\
\hline Vulnerability & Ease of discovery & 1.125 & Relationship & 1.125 \\
Profile & Awareness & 0.750 & Entrusion detection & 0.625 \\
\hline Technical & Loss of confidentiality & 1.125 & Loss of integrity & 1.125 \\
Impact & Loss of availability & 0.875 & Loss of accountability & 1.125 \\
\hline Business & Financial damage & M.C. & Reputation damage & M.C. \\
Impact & Non-compliance & 0.250 & Privacy violation & 0.625 \\
\hline
\end{tabular}

Table 29. Case 2 - Risk modeling results

\begin{tabular}{|l|l|l|l|}
\hline Statistics & Impact Score & Likelihood Score & Risk Score \\
\hline Trials & 100000 & 100000 & 100000 \\
\hline Base Case & 5.1250 & 5.8750 & 3.7637 \\
\hline Mean & 6.3413 & 7.0345 & 5.5758 \\
\hline Median & 6.3750 & 7.1250 & 5.5313 \\
\hline Mode & 6.6250 & 7.1250 & 5.9004 \\
\hline Standard Deviation & 0.5337 & 0.5266 & 0.6279 \\
\hline
\end{tabular}




\begin{tabular}{|l|l|l|l|}
\hline Variance & 0.2849 & 0.2773 & 0.3943 \\
\hline Skewness & 0.0792 & 0.1945 & 0.2514 \\
\hline Kurtosis & 2.29 & 2.30 & 2.74 \\
\hline Coefficient of Variation & 0.0842 & 0.0749 & 0.1126 \\
\hline Minimum & 5.3750 & 6.1250 & 4.1152 \\
\hline Maximum & 7.3750 & 8.1250 & 7.4902 \\
\hline Range Width & 2.0000 & 2.0000 & 3.3750 \\
\hline Mean Std. Error & 0.0017 & 0.0017 & 0.0020 \\
\hline
\end{tabular}

Table 30. Case 2 - Risk modeling assurance table (risk appetite/tolerance)

\begin{tabular}{|l|l|l|l|}
\hline Percentiles & Impact Score & Likelihood Score & Risk Score \\
\hline $0 \%$ & 5.3750 & 6.1250 & 4.1152 \\
\hline $5 \%$ & 5.3750 & 6.1250 & 4.5820 \\
\hline $10 \%$ & 5.6250 & 6.3750 & 4.7734 \\
\hline $15 \%$ & 5.7500 & 6.5000 & 4.8809 \\
\hline $20 \%$ & 5.8750 & 6.5000 & 5.0313 \\
\hline $25 \%$ & 5.8750 & 6.6250 & 5.1211 \\
\hline $30 \%$ & 6.0000 & 6.7500 & 5.1855 \\
\hline $35 \%$ & 6.1250 & 6.7500 & 5.2813 \\
\hline $40 \%$ & 6.1250 & 6.7500 & 5.3789 \\
\hline $45 \%$ & 6.3750 & 7.0000 & 5.4551 \\
\hline $50 \%$ & 6.3750 & 7.1250 & 5.5313 \\
\hline $55 \%$ & 6.3750 & 7.1250 & 5.6465 \\
\hline $60 \%$ & 6.5000 & 7.1250 & 5.6934 \\
\hline $65 \%$ & 6.6250 & 7.1250 & 5.8008 \\
\hline $70 \%$ & 6.6250 & 7.3750 & 5.9004 \\
\hline $75 \%$ & 6.6250 & 7.5000 & 6.0117 \\
\hline $80 \%$ & 6.7500 & 7.5000 & 6.1074 \\
\hline $85 \%$ & 6.8750 & 7.6250 & 6.2227 \\
\hline $90 \%$ & 7.1250 & 7.7500 & 6.4336 \\
\hline $95 \%$ & 7.3750 & 8.1250 & 6.6602 \\
\hline $100 \%$ & 7.3750 & 8.1250 & 7.4902 \\
\hline & & & \\
\hline
\end{tabular}

4.3 Case 3- Online E-Commerce Website Drupal Core 8.0.x Multiple Vulnerabilities In the web based e-commerce application portal chained Drupal vulnerabilities were discovered and validated with Kali Linux using a combination of manual and automated tools. Drupal is prone to multiple vulnerabilities, including security bypass, denial of service, open redirect and information disclosure vulnerabilities. Exploiting these issues could allow an attacker to perform otherwise restricted actions and subsequently view, delete or substitute a link to a file, to cause the affected website to consume memory and CPU resources by blocking file uploads, thus denying service to legitimate users, to redirect users to arbitrary web sites and conduct phishing attacks or to obtain sensitive information that may help in launching further attacks. Drupal Core versions 8.0.x ranging from 8.0.0 and up to and including 8.0.3 are vulnerable. [12] In the Table 31 all the known assumptions for the attributes categories were determined and then using Oracle Crystal Ball we calculated 
the risk profile for the chained Drupal vulnerabilities. The mean impact score is 5.96 (MEDIUM), the mean likelihood score is 7.03 (HIGH) and the mean risk score is 5.24 (MEDIUM), as presented in the Table 32. Although the quantitative analysis calculated a mean risk score of 5.96, using the percentiles assurance values from Table 33 the organization can eliminate or keep a level of uncertainty depending on their risk appetite. In this case the organization had a low risk appetite given the importance of their e-commerce application portal to their business model and business reputation and they wanted $100 \%$ assurance, meaning that the vulnerability risk score was officially 7.10 (HIGH) with an impact score of $7.00(\mathrm{HIGH})$ and a likelihood score of 7.10 (HIGH). The results were presented to the organization's management who allocated the resources, time and budget to repair the vulnerability.

Table 31. Case 3 - Risk modeling assumptions

\begin{tabular}{|c|l|c|l|c|}
\hline $\begin{array}{c}\text { Risk } \\
\text { Component }\end{array}$ & Risk Subcomponent & Score & Risk Subcomponent & Score \\
\hline $\begin{array}{c}\text { Threat Actor } \\
\text { Profile }\end{array}$ & Skill Level & M.C. & Motive & M.C. \\
\hline Vulnerability & Ease of discovery & 1.125 & Relationship & 1.125 \\
Profile & Awareness & 0.750 & Ease of exploit & 0.375 \\
\hline Iechnical & Loss of confidentiality & 0.625 & Loss of integrity & 1.125 \\
Impact & Loss of availability & 0.875 & Loss of accountability & 0.875 \\
\hline Business & Financial damage & M.C. & Reputation damage & M.C. \\
Impact & Non-compliance & 0.250 & Privacy violation & 0.875 \\
\hline
\end{tabular}

Table 32. Case 3 - Risk modeling results

\begin{tabular}{|l|l|l|l|}
\hline Statistics & Impact Score & Likelihood Score & Risk Score \\
\hline Trials & 100000 & 100000 & 100000 \\
\hline Base Case & 4.7500 & 5.8750 & 3.4883 \\
\hline Mean & 5.9676 & 7.0363 & 5.2487 \\
\hline Median & 6.0000 & 7.1250 & 5.2070 \\
\hline Mode & 5.7500 & 7.1250 & 5.1211 \\
\hline Standard Deviation & 0.5343 & 0.5273 & 0.6143 \\
\hline Variance & 0.2855 & 0.2780 & 0.3774 \\
\hline Skewness & 0.0822 & 0.1899 & 0.2660 \\
\hline Kurtosis & 2.29 & 2.30 & 2.74 \\
\hline Coefficient of Variation & 0.0895 & 0.0749 & 0.1170 \\
\hline Minimum & 5.0000 & 6.1250 & 3.8281 \\
\hline Maximum & 7.0000 & 8.1250 & 7.1094 \\
\hline Range Width & 2.0000 & 2.0000 & 3.2813 \\
\hline Mean Std. Error & 0.0017 & 0.0017 & 0.0019 \\
\hline
\end{tabular}

Table 33. Case 3 - Risk modeling assurance table (risk appetite/tolerance)

\begin{tabular}{|l|l|l|l|}
\hline Percentiles & Impact Score & Likelihood Score & Risk Score \\
\hline $0 \%$ & 5.0000 & 6.1250 & 3.8281 \\
\hline $5 \%$ & 5.0000 & 6.1250 & 4.2832 \\
\hline $10 \%$ & 5.2500 & 6.3750 & 4.4531 \\
\hline $15 \%$ & 5.3750 & 6.5000 & 4.5938 \\
\hline $20 \%$ & 5.5000 & 6.5000 & 4.6895 \\
\hline
\end{tabular}




\begin{tabular}{|l|l|l|l|}
\hline $25 \%$ & 5.5000 & 6.6250 & 4.7871 \\
\hline $30 \%$ & 5.6250 & 6.7500 & 4.8809 \\
\hline $35 \%$ & 5.7500 & 6.7500 & 4.9805 \\
\hline $40 \%$ & 5.7500 & 6.7500 & 5.0625 \\
\hline $45 \%$ & 6.0000 & 7.0000 & 5.1211 \\
\hline $50 \%$ & 6.0000 & 7.1250 & 5.2070 \\
\hline $55 \%$ & 6.0000 & 7.1250 & 5.3008 \\
\hline $60 \%$ & 6.1250 & 7.1250 & 5.3789 \\
\hline $65 \%$ & 6.2500 & 7.1250 & 5.4688 \\
\hline $70 \%$ & 6.2500 & 7.3750 & 5.5664 \\
\hline $75 \%$ & 6.3750 & 7.5000 & 5.6777 \\
\hline $80 \%$ & 6.3750 & 7.5000 & 5.7891 \\
\hline $85 \%$ & 6.5000 & 7.6250 & 5.9063 \\
\hline $90 \%$ & 6.7500 & 7.7500 & 6.0762 \\
\hline $95 \%$ & 7.0000 & 8.1250 & 6.3281 \\
\hline $100 \%$ & 7.0000 & 8.1250 & 7.1094 \\
\hline
\end{tabular}

\section{Conclusions}

The experimental results show that the proposed web application risk modeling method can deliver reliable results related to quantitative risk assessment that enable users to take better decisions on how to manage their web applications risks. The available literature on this particular subject is very limited, most of the research revolving around comparative studies between risk management frameworks like the Core Unified Risk Framework [13] or on improving the IT risk assessment model proposed by NIST [14]. Since the most widespread risk assessment model relies on the annual loss expectancy (ALE) and single loss expectancy (SLE) [15] formulas that in practice involve accepting a high level of uncertainty most organizations are relying solely on the qualitative risk assessment models and are classifying risk in ranges from low to high or critical. The quantitative risk modeling method described in this research is considerably reducing the guess work and uncertainty from the risk assessment activities while providing sufficient flexibility to users to model risks according to their risk posture (appetite and tolerance) by using high rounds of Monte Carlo simulations on 1 and up to 16 risk attributes.

\section{References}

[1] World Biggest Data Breaches. Available: http://www.informationisbeauti-

ful.net/visualizations/worlds-biggestdata-breaches-hacks/

[2] EY Global Information Security Survey 2016. Available: http://www.ey.com/gl/en/services/advisory/ey-global-information-security-survey-2016

[3] OWASP Risk Rating Methodology. Available: https://www.owasp.org/index.php/OWASP_Risk_Rating_Methodology

[4] OWASP Foundation. Available: https://www.owasp.org/index.php/Main_Page

[5] Oracle Crystal Ball. Available: http://www.oracle.com/us/products/applications/crystalball/overview/index.html

[6] Acunetix Web Vulnerability Scanner. Available: https://www.acunetix.com/

[7] Kali Linux. Available: https://www.kali.org/

[8] Rapid 7 Metasploit. Available: https://www.metasploit.com/

[9] SQLMAP. Available: http://sqlmap.org/

[10] SQL Injection Vulnerability. Available: https://www.acunetix.com/vulnerabilities/web/sql-injection

[11] Joomla remote code execution. Available: https://www.acunetix.com/vulnerabilities/web/joomla-core-remote-code-execution-1-5-0-3-4-5 
[12] Drupal core multiple vulnerabilities. Available: (https://www.acunetix.com/vulnerabilities/web/drupalcore-8-0-x-multiple-vulnerabilities-8-0-0$8-0-3$ )

[13] Core Unified Risk Framework. Available: https://link.springer.com/article/10.1007/s10207-017-0382-0

[14] NIST Guide for Conducting Risk Assess-

ments. Available: http://nvlpubs.nist.gov/nistpubs/Legacy/SP/nistspecialpublication80030r1.pdf

[15] SANS Quantitative Risk Analysis StepBy-Step. Available: https://www.sans.org/readingroom/whitepapers/auditing/quantitativerisk-analysis-step-by-step-849

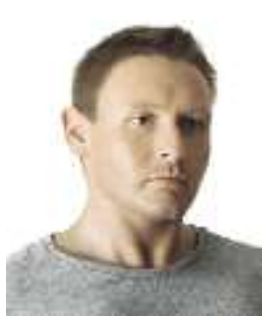

Sergiu SECHEL has graduated the Faculty of Automation and Applied Computer Sciences in 2009. He is a PhD Candidate in Economy Informatics at the Bucharest University of Economic Sciences and an Advisory Manager at EY (Ernst \& Young). His areas of research are cybersecurity, audit, risk management and malware research. 